\title{
PODER ANTIOXIDANTE Y DAÑO CELULAR EN EL CARCINOMA DE PRÓSTATA
}

\author{
José Daniel López Laur',2, María Abud', Constanza López Fontana², Joaquín Silva', Yolanda \\ Cisella', Rafael Pérez Elizalde² y Alba Ortiz?
}

'Cátedra de Química Urológica y Cátedra de Química Biológica. Facultad de Ciencias Médicas. Universidad Nacional de Cuyo. Mendoza. Argentina.

¿Laboratorio de Enfermedades Metabólicas y Cáncer. Facultad de Farmacia y Bioquímica. Universidad Juan Agustín Maza. Mendoza. Argentina.

Resumen.- OBJETIVOS: Determinar y comparar el poder antioxidante, el daño celular por peroxidación lipídica y el daño de membrana en pacientes normales y en aquellos con carcinoma de próstata y así, determinar la capacidad antitumoral.

MÉTODO: La población estuvo constituida por 19 pacientes con PSA total elevado, mayor de $4 \mathrm{ng} / \mathrm{ml}$ con un mínimo de 10 biopsias transrectales ecodirigidas y 10 sujetos normales como grupo control. En todos los casos se efectuó historia clínica, tacto rectal, examen de PSA y analítica de rutina; además de técnicas espectrofotomé- tricas para medir poder antioxidante. El daño de membrana se midió a través de la malonildialdehido (MDA) y el daño celular mediante la glutation pexoxidasa.

RESULTADOS: Del total de 19 casos: 6 (3 1,5\%) presentaron carcinoma de próstata 14 con Gleason 7; 1 con Gleason 6 y 1 con Gleason 8); 8 presentaron histopatológicamente hiperplasia benigna de próstata con componente de prostatitis crónica ; 3 pacientes con hiperplasia prostática y atrofia glandular; y 2 casos con hiperplasia benigna de próstata aislada.Los 10 sujetos considerados controles presentaron un rango de normalidad en las todas las determinaciones. Mientras que en los pacientes estudiados, el poder antioxidante fue menor a $1,30 \mathrm{mmol} / \mathrm{L}$ en 13 casos; supero $1,77 \mathrm{mmol} / \mathrm{L}$ en 4 sujetos y 2 persistieron en valores normales. La MDA se encontró aumentada en 15 pacientes y 4 fueron normales siendo aquellos que no tenían prostatitis crónica en su histología. Cuatro de los pacientes con carcinoma de próstata presentaron 4 un poder antioxidantes disminuido menor a 0.90 , estando en relación a la arquitectura glandular, ya que los de Gleason 8 y 7 no superaron $0,58 \mathrm{mmol} / \mathrm{I}$. Dos pacientes con carcinoma de próstata de Gleason más bajo presentaron valores mínimos normales.

CONCLUSIONES: Los pacientes con carcinoma de próstata o procesos reactivos, como la prostatitis crónica y la prostatitis atrófica, presentan una disminución del poder antioxidante y un aumento de la peroxidación lipídica.

Palabras clave: Poder antioxidante. Estrés oxidativo. Carcinoma de próstata. Malonildialdehido. 
Summary.- OBJECTIVES: To determine and compare the antioxidant power, cellular damage by lipidic peroxidation, and membrane damage in normal patients and patients with prostate cancer, so determining the antitumoral capacity.

METHODS: The study population included 19 patients with elevated total PSA, greater than $4 \mathrm{ng} / \mathrm{ml}$, with a minimum of 10 ultrasound guided transrectal biopsies, and 10 normal subjects as control group. In all cases, medical history, rectal digital examination, PSA determination and routine analyses were performed in addition to spectrophotometric tests to measure the antioxidant power. Membrane damage was measured by detemination of malonyldialdehyde (MDA) and cellular damage by glutathione peroxidase.

RESULTS: From a total of 19 cases: 6 (31.5\%) presented prostate cancer (4 Gleason 7; 1 Gleason 6 and 1 Gleason 81; 8 presented histological benign prostatic hyperplasia with a component of chronic prostatitis; 3 patients prostatic hyperplasia and glandular atrophy; and 2 cases isolated benign prostatic hyperplasia. All 10 control patients presented values within normal range in all determinations, whereas study patients showed antioxidant power $<1.3 \mathrm{mmol} / \mathrm{l}$ in 13 cases; higher than $1.77 \mathrm{mmol} / \mathrm{l}$ in 4 and normal values in 2 . MDA was elevated in 15 patients and normal in 4, all of them without histological chronic prostatitis. Four of the patients with prostate cancer presented a diminished antioxidant power below 0.90, with a relationship with glandular architecture, because those with Gleason 8 and 7 did not exceed $0.58 \mathrm{mmol} / \mathrm{l}$. Two patients with prostate cancer with a lower Gleason score presented normal minimal values.

CONCLUSIONS: Patients with prostate cancer or reactive processes, such as chronic prostatitis or atrophic prostatitis, present a decreased antioxidant power and an increase of lipidic peroxidation.

Keywords: Antioxidant power. Oxidative stress. Prostate cancer. Malonylaldehyde.

\section{INTRODUCCIÓN}

El oxígeno molecular $\left(\mathrm{O}_{2}\right)$ posee orbitales completos o apareados que le confieren estabilidad molecular y además, como es liposoluble, facilita su movilización a través de membranas celulares y organelas y con sólo 0,2 atmósferas de presión parcial de oxígeno realiza el metabolismo oxidativo tisular.

Un aspecto que resalta la enorme importancia de la incidencia del cáncer de próstata, es su relación con el estrés oxidativo.
Como sabemos, los procesos malignos de proliferación celular están relacionados a la capacidad de producir estrés oxidativo y peroxidación lipídica de la membrana celular, alterando el ADN y sus mecanismos de replicación celular.

El conocimiento de cuánto es el poder antioxidante que posee un paciente o bien el grado de daño celular, nos permitirá un mejor manejo de su respuesta celular, evaluando sus propiedades anticarcerígenas y su facilidad para poner en juego mecanismos reparadores.

\section{OBJETIVO}

Determinar y comparar el poder antioxidante, $y$ el daño de membrana en pacientes normales y en aquellos con carcinoma de próstata y así, determinar la capacidad antitumoral.

\section{MATERIAL Y MÉTODOS}

La población estuvo constituida por 19 sujetos con PSA total elevado, mayor de $4 \mathrm{ng} / \mathrm{ml}$ y biopsiados con un mínimo de 10 biopsias transrectales ecodirigidas de próstata y 10 sujetos considerados normales como grupo control. El rango de edad fue de 50 a 77 años, sin dieta previa, ni con ingesta de antioxidante externo medicamentoso específico.

En todos los casos se efectuó una historia clínica, tacto rectal, examen de PSA y analítica de rutina, además de técnicas bioquímicas espectrofotométricas estandarizadas para medir poder antioxidante.

El daño de membrana fue evaluado con la determinación de malonildialdehido (MDA).

- El poder ANTIOXIDANTE TOTAl, se basa en el ABTS 82,2 azino-di-(3-etibenzoatolin sulfonato) que se incuba con peroxidasa (metamioglobina) y $\mathrm{H}_{2} \mathrm{O}_{2}$ para dar el radical ABTS+. Este radical presenta una coloración verde azulada relativamente estable, que se mide a $600 \mathrm{~nm}$.

La presencia de antioxidantes en la muestra produce una supresión de esta coloración, siendo esta supresión proporcional a la concentración de antioxidantes. Valores normales: 1,30-1,77mmol/l.

- La determinación de la malonil- dialdehido (MDA), se basa en la condensación de una molécula de MDA con dos moléculas de $\mathrm{N}$-metil-2-fenilindol en acetonitrilo, dando un cromóforo estable con máxi- 


\section{Resultado de las Biopsias}

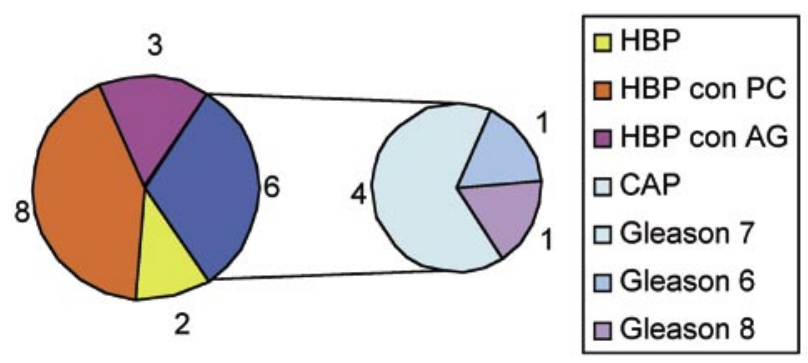

FIGURA 1. Resultado de las biopsias.

ma absorción a $586 \mathrm{~nm}$. Valores Normales: 0,5-0,8 Umol/l.

\section{RESULTADOS}

El resultado de las biopsias realizadas mostró que de un total de 19 casos: $6(31,5 \%)$ presentaron carcinoma de próstata $(4$ con Gleason $7 ; 1$ con Gleason 6 y 1 con Gleason 8); 8 presentaron histopatológicamente hiperplasia benigna de próstata con componente de prostatitis crónica; 3 pacientes con hiperplasia prostática y atrofia glandular; y 2 casos con hiperplasia benigna de próstata aislada (Figura 1).

Los 10 sujetos considerados controles presentaron un rango de normalidad en las todas las determinaciones. Mientras que en los pacientes estudiados, el poder antioxidante fue menor a 1,30 $\mathrm{mmol} / \mathrm{L}$ (VN 1,30-1,77mmol/L) en 13 casos; superó

\section{Poder antioxidante (VN :1,3 - 1,77 mmol/l)}

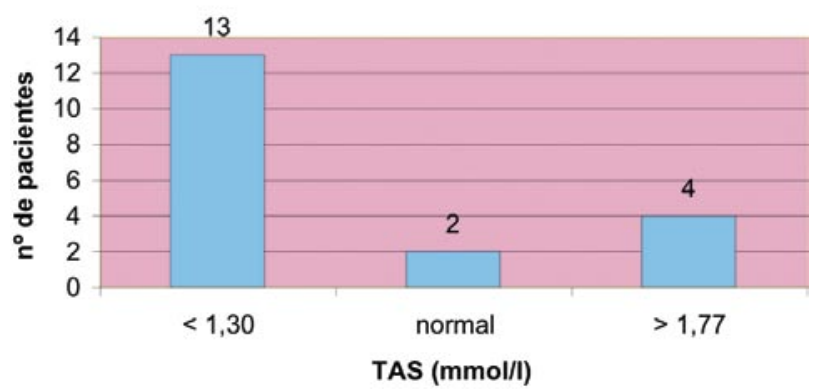

FIGURA 2. Valores de poder antioxidante de los sujetos estudiados.
$1,77 \mathrm{mmol} / \mathrm{l}$ en 4 sujetos y 2 persistieron en valores normales (Figura 2). Cuatro de los pacientes con carcinoma de próstata presentaron un poder antioxidante disminuido menor a $0.90 \mathrm{mmol} / \mathrm{l}$, estando en relación a la arquitectura glandular, ya que los de Gleason 8 y 7 no superaron 0,58mmol/l. Dos pacientes con carcinoma de próstata de Gleason más bajo presentaron valores mínimos normales.

La MDA, cuyo rango varia de 0,5 a $0,8 \mathrm{Umol} / \mathrm{l}$, presentaron un aumento en 15 pacientes y 4 fueron normales siendo aquellos que no tenían prostatitis crónica en su histología (Figura 3).

\section{DISCUSIÓN}

El cáncer de próstata puede ser prevenido y por ello es indispensable la quimioprevención y la adopción de medidas estratégicas para evitarlo o modificar su curso $(1,2)$.

El estrés oxidativo genera una alteración en la molécula de ADN y no permite la apoptosis adecuada afectando el ciclo celular normal y favoreciendo el desarrollo de la Carcinogénesis.

Un radical libre se define como una especie química con capacidad de existencia independiente que posee un orbital con un electrón desapareado. Puesto que los electrones son más estables cuando están apareados, los radicales libres son especies altamente reactivas, y una vez formadas reaccionan con cualquier molécula situada en la proximidad. A nivel fisiológico, los radicales libres más importantes son especies derivadas del oxígeno, tales como el anión superóxido, el radical hidroxilo, el óxido nítrico, el radical peróxido, el radical hidroperoxilo y el oxígeno singulete. Además de los radicales libres, existen

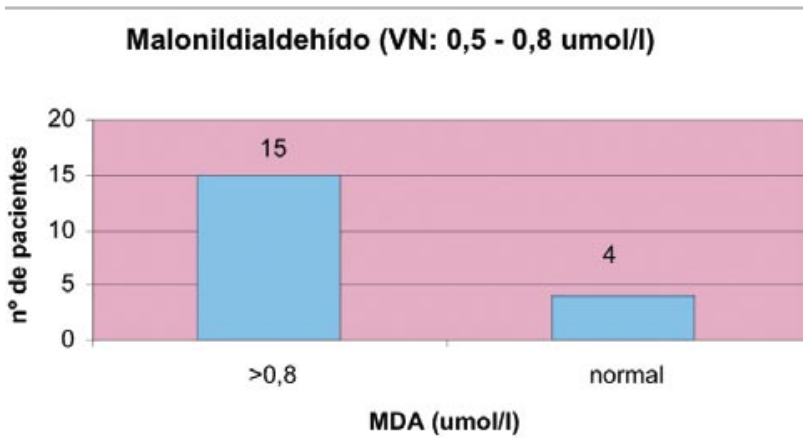

FIGURA 3. Valores de malonildialdehído de los sujetos estudiados. 
otras especies derivadas del oxígeno no radicalarias que se conocen como especies de oxígeno reactivas y que pueden ser oxidantes en ciertas condiciones, como por ejemplo el peróxido de hidrógeno, el ácido hipocloroso y el ozono. Se podría decir que es una producción de radicales libres accidental (Tabla I).

En el organismo humano, los radicales libres se producen durante el metabolismo aeróbico celular, a través de reacciones enzimáticas, entre las que se incluyen la cadena respiratoria, el proceso de fagocitosis, la síntesis de prostaglandinas y el sistema citocromo P-450. Pero los radicales libres también se pueden generar a partir de reacciones no enzimáticas como las que se producen por reacción del oxígeno con compuestos orgánicos o las iniciadas por radiaciones ionizantes. Es así que el organismo está expuesto a agentes oxidantes ambientales como el ozono, óxidos de nitrógeno, humo de tabaco, gases del motor de los vehículos. Aunque la mayoría de radicales libres que se forman en los sistemas biológicos lo hacen mediante reacciones enzimáticas que están implicadas en el mantenimiento de las funciones del organismo, los radicales formados por procesos no enzimáticos y también los que escapan de las reacciones enzimáticas pueden producir modificaciones aleatorias $y$, en último término, daño celular. Debido a su elevada reactividad, los radicales libres formados son capaces de interaccionar con diversas estructuras celulares: a través de la unión covalente a las enzimas o receptores, modificando su actividad; mediante la unión a ciertos componentes de la membrana, alterando la estructura y función de la misma e interfiriendo en los procesos de transporte; iniciando la peroxidación lipídica, con graves consecuencias estructurales y funcionales, o causando daño sobre el ADN.

Debido a su potencial para dañar los sistemas biológicos principales, se considera que los radicales libres son factores importantes que contribu-

TABLA I. ESPECIES REACTIVAS DE OXÍGENO (EROS).

\begin{tabular}{|l|l|}
\hline Radicales Libres & No Radicales \\
\hline $\mathrm{ROO}^{\circ}$ Radical Peróxido & $\mathrm{H}_{2} \mathrm{O}_{2}$ Peróxido de hidrógeno \\
\hline $\mathrm{OH}^{\circ}$ Radical Hidroxilo & $\mathrm{I}_{2}$ Oxígeno Singlete \\
\hline $\mathrm{O}_{2}^{--}$Anión Superóxido & \\
\hline
\end{tabular}

yen al desarrollo de enfermedades crónicas como la enfermedad cardiovascular y el cáncer, en las que el estrés oxidativo se presenta como un fenómeno secundario que puede agravar el curso de las mismas. Entre las múltiples teorías sobre el envejecimiento, una de las que actualmente recibe mayor atención es la teoría de los radicales libres, postulada por Harman, que señala los radicales libres como agentes responsables del fenómeno de la senescencia y el cáncer $(3,4)$.

Los sistemas biológicos han desarrollado toda una serie de mecanismos de defensa o antioxidantes contra los efectos perjudiciales de los radicales libres. En este sentido, la cadena transportadora de electrones que se encuentra en la mitocondria sería la primera arma frente a los radicales libres, ya que permite la utilización del oxígeno para la obtención de energía evitando al máximo la pérdida de intermediarios radicalarios. Los sistemas de defensa propiamente dichos están formados por compuestos de tipo enzimático que secuestran las especies de oxígeno reactivas, como la superóxido dismutasa (SOD), catalasa, glutatión peroxidasa, glutatión reductasa y glutatión-S-transferasa. De todos estos sistemas enzimáticos, los más importantes en la inactivación de los radicales libres a nivel intracelular son la superóxido dismutasa, la catalasa y la glutatión peroxidasa. Asimismo, existen una serie de compuestos de tipo no enzimático, que también tienen un papel protector relevante frente a los radicales libres, como el glutatión, vitaminas y provitaminas, ceruloplasmina, selenio y otros elementos necesarios para la función de los enzimas antioxidantes. Estos compuestos actúan de forma complementaria y sinérgica. En el caso de que los radicales libres lleguen a producir daño sobre alguna estructura celular, los seres vivos disponen de diversos mecanismos de reparación entre los que se incluyen las ligasas, polimerasas, nucleasas, proteinasas y fosfolipasas, que subsanan el daño causado por los radicales libres (5-7).

El estrés oxidativo es una condición celular asociada a un disbalance entre la producción de EROS (especies reactivas de oxígeno) y la capacidad de "limpiar" de agentes oxidante el medio celular (8).

El equilibrio entre las sustancias prooxidantes y los sistemas antioxidantes de defensa en el organismo se define como el estado antioxidante. Lo que se conoce como estrés oxidativo es consecuencia de un grave desequilibrio en favor de un estado de oxidación, a causa de una producción excesiva de especies de oxígeno reactivas o de una disminución de los sistemas de defensa antioxidante. Puesto que el estrés oxidativo severo puede llegar a producir daño 
celular, su prevención es importante para el mantenimiento de una buena salud.

Los factores dietéticos influyen tanto sobre el sistema antioxidante como en la producción de radicales libres y especies de oxígeno reactivas, mientras que los demás influyen sólo en la producción de radicales libres y especies de oxígeno reactivas.

\section{Antioxidantes}

Algunos trabajos realizados por Cutler, indican que existe una correlación positiva entre la longevidad máxima de una especie y la concentración de determinados antioxidantes fisiológicos como la enzima superóxido dismutasa (SOD), el ácido úrico, la vitamina $\mathrm{E}$ y los carotenoides. Diversos estudios científicos han revelado que la suplementación de la dieta con antioxidantes no tiene ningún efecto sobre la esperanza de vida. Si bien parece que estas estrategias no tienen el menor efecto sobre la longevidad máxima, se cree que sí pueden tener un efecto beneficioso sobre el envejecimiento de ciertos sistemas o que pueden tener un efecto positivo sobre la longevidad media, gracias a su papel beneficioso en ciertas enfermedades crónicas.

El daño oxidativo causado por los radicales libres es una característica de la mayoría de enfermedades que afectan al hombre, entre las que se encuentran la enfermedad cardiovascular y el cáncer.

Es posible que algunas reacciones radicalarias que se producen en el organismo sean iniciadores o promotores de determinados tipos de cáncer. El paralelismo entre la incidencia de cáncer y la edad puede estar relacionado con un mayor número de reacciones radicalarias en el organismo con el tiempo, unido a la progresiva disminución de la capacidad del sistema inmune para eliminar las células cancerosas. A causa de la relación entre los procesos oxidativos y el cáncer, muchos estudios han evaluado el papel de los antioxidantes de la dieta en el desarrollo de esta enfermedad. En este sentido, los micronutrientes de la dieta con propiedades antioxidantes podrían ser considerados como antipromotores del cáncer, mientras que una deficiencia de antioxidantes como la vitamina $\mathrm{E}$, el betacaroteno, el ácido ascórbico o vitamina $C$ y el selenio en la dieta podría contribuir al desarrollo del cáncer y de otras enfermedades degenerativas (2-4). Se han sugerido diversos mecanismos por los cuales las sustancias anticancerígenas pueden interferir en la formación de tumores:

a) Evitan la iniciación del tumor gracias a sus propiedades antioxidantes y secuestradores de radicales libres. b) Evitan la formación de agentes mutágenos.

c) Aumentan la eliminación hepática de toxinas vía citocromo P-450.

d) Inhiben la etapa de iniciación de un tumor alterando las funciones celulares.

e) Pueden captar las formas activas de los carcinógenos evitando así que lleguen a los lugares diana.

f) Pueden aumentar los sistemas de defensa del organismo.

g) Pueden inhibir o reducir la progresión de un cáncer ya iniciado.

Los estudios epidemiológicos han encontrado una asociación entre una ingesta elevada de alimentos ricos en vitamina $C$, o betacaroteno y un menor riesgo de ciertos tipos de cáncer. Otros estudios han demostrado en cambio una relación entre niveles séricos bajos de vitamina $\mathrm{E}$ y mayor riesgo de cáncer. Los resultados de un estudio de intervención llevado a cabo en Finlandia mostraron una disminución en la incidencia de cáncer de próstata y de colon y recto en sujetos fumadores después de 5-8 años de suplementación con dosis elevadas de alfatocoferol, pero un aumento en la incidencia de cáncer de estómago. También el estudio Linxian, realizado en China, en el que se utilizó una combinación de betacaroteno, vitamina $\mathrm{E}$ y selenio a dosis nutricionales, ha demostrado un efecto positivo de la suplementación con antioxidantes sobre la mortalidad global y la incidencia de cánceres $(6,9)$. Otros estudios sobre el efecto de distintos antioxidantes en varios tipos de cáncer también han obtenido resultados negativos. La ingesta de suplementos vitamínicos debe ser tomada con precaución por el momento, aunque sí parece razonable recomendar un aumento de alimentos ricos en vitaminas en la dieta. Se está estudiando el papel de los elementos traza en los principales tipos de cáncer. Algunos estudios han encontrado una relación positiva entre los niveles de hierro en el organismo y el riesgo de cáncer (10).

La ingesta o suplementacion de Selenio y Zinc son factores esenciales para aumentar el poder antioxidante del individuo y favorecer la apoptosis. $(11,12)$.

Lo mismo podemos decir para los licopenos que producen, aportados exógenamente, un descenso del PSA del orden del $39.11 \%$. Los niveles del mismo alcanza su plateau a los 3 meses, siendo útiles aún cuando se haya hecho ya un tratamiento definitivo $(7,13)$. 
El análisis de nuestros pacientes muestra como aspecto interesante una disminución del poder antioxidante en gran parte de los enfermos y curiosamente permaneció normal en dos de ellos que interpretamos haya habido un suplemento dietario o medicamentoso que no fue especificado el poder antioxidante puede elevarse durante un día por razones de difícil determinación.

El daño de membrana que medimos por MDA que además nos informa sobre el daño celular producidas por las ERO es un índice de peroxidación lipídica y que tiene una mayor sensibilidad en el diagnóstico de lesión celular ya que solo fue normal en cuatro pacientes que no presentaban en su anatomía patológica que no fuese hiperplasia prostática benigna.

Estos resultados revelan una alteración en el índice de oxidación lipídica con cambios concomitantes en el sistema de defensa antioxidante en pacientes con cáncer de próstata comparado con la hiperplasia benigna de próstata (BPH) $(5,14)$.

Permite distinguir pacientes con status antioxidante adecuado para su reemplazo entre HBP y cáncer de próstata (14).

Nosotros creemos que el balance prooxidante-antioxidante puede llevar a un incremento del daño oxidativo y consecuentemente jugar un rol importante en la carcinogénesis prostática.

De lo anterior se desprende que la quimioprevención es una alternativa y una luz para el tratamiento del cáncer prostático y sobre todo para mejorar sus mecanismos de prevención $(6,9,15)$.

En artículos anteriores desarrollados por nosotros sobre dosaje de glutatiónperoxidasa y selenio, aporte de licopeno y modificaciones del PSA, como el de otros carotenoides y elementos antioxidantes, fitoestrógenos demuestran que los mismos pueden alterar la historia natural del cáncer de próstata como escribe Srivastava AR y cols. $(6,7,9,16)$.

El aumento de la capacidad antioxidante y las modificaciones en plasma de los niveles de malonildialdehido o MDA constituyen uno de los índices valorables para la determinación de la situación antitumoral de un paciente.

\section{CONCLUSIONES}

El presente estudio permite concluir que los pacientes con carcinoma de próstata $\circ$ procesos reactivos, como la prostatitis crónica y la prostatitis atrófica, presentan una disminución del poder antioxidante y un aumento de la peroxidación lipídica.

\section{AGRADECIMIENTO}

Agradecemos la colaboración de los becarios del Laboratorio de Investigación en Enfermedades Metabólicas y Cáncer: Gabriela Recalde, Nicolás Di Milta, Eugenia Maselli y Cristina Vanrell.

\section{BIBLIOGRAFÍA Y LECTURAS RECOMENDADAS (*lectura de interés $y^{* *}$ lectura fundamental)}

*1. KLEIN, A.: "Can prostate cancer be prevent?". Nat. Clin. Pract. Urol., 2: 2431, 2005.

**2. FAIR, W.; FLESHNER, N.E.; HESTON W.: "Cancer of the prostate: A nutritional disease?". Urology, 50: 840, 1997.

*3. YILMAZ, M.; SAGLAM, K.; SONMEZ, A. y cols.: "Antioxidant system activation in prostate cancer". Biol. Trace. Elem. Res., 98: 13, 2004.

4. SOLE BALCELLS, F.: "El urólogo como generalista de la gente mayor". Actas Urol. Esp., 30: 103, 2006.

5. OZMEN, H.; ERULAS, F.A.; KARATAS, F. y cols.: "Comparision of de concentration of trace metals ( $\mathrm{Ni}, \mathrm{Zn}, \mathrm{Co}, \mathrm{Cu}$ and $\mathrm{Se}$ ), $\mathrm{Fe}$, vitamins $\mathrm{A}$, $\mathrm{C}$ and $\mathrm{E}$, and lipid peroxidation in patients with prostate cancer". Clin. Chem. Lab. Med., 44: 175, 2006.

*6. LOPEZ LAUR, J.D.; LOPEZ FONTANA, C.; LOPEZ FONTANA, G. y cols.: "Modificación del PSA con el aporte de licopeno". Rev. Medica Universitaria, 2:1, 2006.

*7. LOPEZ LAUR, J.D.; LOPEZ FONTANA, C.: "Aporte de licopenos y modificaciones del PSA". Rev. Arg. Urol., 70: 153, 2005.

8. ASHOK, A.; PRABAKARAN, S.; SIKKA, S.: "Clinical relevance of oxidative stress in patients with male factor infertility: Evidence based analysis". AUA update Series, 26, 2007.

*9. LOPEZ FONTANA, C.; LOPEZ LAUR, J.D.: "Nutrición y cáncer de próstata". Edit. Aguirre. Mendoza, Argentina, 2001.

10. CANBY-HAGINO, E.; THOMPSON, I.: "Mechanism of disease: Prostate cancer, a model for cancer chemoprevention in clinical practice". Nat. Clin. Pract. Oncol., 2: 255, 2005.

**11. COMBS, G.: "Status of selenium in prostate cancer prevention”. Brit. J. Cancer, 19: 195, 2004. 
12. LOPEZ LAUR, J.; ABUD, M.; ORTIZ, A. y cols.: "Glutation peroxidasa y selenio en pacientes con alto riesgo de cancer de próstata". Actas Cong. Arg. Urol. Bs. As, 2005.

**13. CLARK, P.; HALL, M.C.; BORDEN, L.S. Jr. y cols.: "Phase I-II prospective dose escalating trial of licopene in patients with biochemical relapse of prostate cancer alter definitive local therapy". Urology, 67: 1257, 2006.

*14. AYDIN, A.; SAYAL, A.; EKEN, A. y cols.: "Oxidative stress and antioxidant status in non metastatic prostate cancer and benign prostatic hyperplasia”. Clin. Biochem., 39: 179, 2006.

15. FUJIMOTO, N.; NOMURA, M.; MATSUMOTO, T.: "Current and future status of prostate cancer chemoprevention". Expert. Rev. Anticancer Ther., 6: 59, 2006.

16. SRIVASTAVA, A.; DALELA, D.: "Prostate cancer: Altering the natural history by dietary changes". Nat. Med. J. India, 17: 248, 2004. 